\title{
MARKOV PROPERTY OF EXTREMAL LOCAL FIELDS
}

\author{
N. DANG-NGOC ${ }^{1}$ AND G. ROYER ${ }^{2}$
}

\begin{abstract}
We show that extremal local field on $(E, \mathcal{E})^{T}$, with $T=\mathbf{Z}$ or $\mathbf{R}$ and $(E, \mathcal{E})$ standard, possesses the Markov property. This result generalizes that of $\mathrm{F}$. Spitzer in the case $T=\mathbf{Z}, E$ countable and a result of $\mathrm{G}$. Royer and $M$. Yor on extremal measures associated to certain diffusion processes.
\end{abstract}

I. Introduction. Let $T=\mathbf{R}$ or $\mathbf{Z}$, let $(E, \mathcal{E})$ be a standard Borel space, $(\Omega, \mathcal{Q}, P)$ a probability space and $\left(X_{t}\right)_{t \in T}$ a stochastic process taking values in $E$. If $\Lambda$ is a subset of $T$, we note $\mathbb{Q}_{\Lambda}$ the sub- $\sigma$-algebra of $\mathscr{Q}$ generated by $X_{t}, t \in \Lambda$.

The process $\left(X_{t}\right)$ is called a local Markov process (or local Markov field) if for every $a<b$ in $T$, every positive $\mathbb{Q}_{[a, b]}$-measurable function $f$ we have

$$
E\left[f \mid \mathscr{Q}_{-\infty, a} \bigcup b,+\infty l\right]=E\left[f \mid \mathscr{Q}_{\{a, b\}}\right] \quad P \text {-a.s. }
$$

The process $\left(X_{t}\right)$ is called a Markov process (or Markov field) if for every $t$ in $T$, every positive $\mathbb{Q}_{t,+\infty}$ measurable function $f$ we have

$$
E\left[f \mid \mathscr{Q}_{-\infty, t]}\right]=E\left[f \mid \mathbb{Q}_{t}\right] \quad P \text {-a.s. }
$$

The property (0) is called local Markov property, the property (1) is called Markov property, it is known that Markov property implies local Markov property (see for example [1]) but the converse is false (cf. [4], [5]).

The purpose of this paper is to show that under quite general conditions the local Markov property and the hypothesis $\mathbb{Q}_{\infty}=\{\varnothing, \Omega\} P$-a.s. imply the Markov property, where $\mathfrak{U}_{\infty}=\cap_{t \in T} \mathbb{Q}_{1, \infty}$ is the asymptotic $\sigma$-algebra of the process (we note similarly $\mathbb{Q}_{-\infty}=\cap_{t \in T} \mathbb{Q}_{-\infty, t]}$ ).

This result has been proved under the hypotheses $E$ countable and $T=\mathbf{Z}$ by F. Spitzer (cf. [5]). In the case $E=\mathbf{R}, T=\mathbf{R}, \mathrm{G}$. Royer and M. Yor have proved this result for certain diffusion processes associated to onedimensional quantum fields (cf. [4]).

\section{Sufficient conditions for Markov property.}

Notations. We take the path space $(\Omega, \mathscr{Q})=(E, \mathcal{E})^{T}, X_{t}(\omega)=\omega_{t}, \forall \omega \in$ $E^{T}, \forall t \in T$. If $\mu$ is a probability on $(\Omega, \mathcal{Q})$, and $\Lambda \subset T$, we note $\mu_{\Lambda}$ the

\footnotetext{
Received by the editors March 28, 1977 and, in revised form, June 13, 1977.

AMS (MOS) subject classifications (1970). Primary 60J05, 60J25, 60K35.

Key words and phrases. Markov field, local Markov field, Markov process, Markov property, Gibbs state.

${ }^{1}$ Université de l'Etat à Mons (Belgique) et Equipe de Recherche no. 1 "Processus stochastiques et applications" associée au C.N.R.S. (France).

${ }^{2}$ Equipe de Recherche associée au C.N.R.S. no. 294.
} 
restriction of $\mu$ on $\mathbb{Q}_{\Lambda}$ and $E_{\mu}\left[g \mid \mathbb{Q}_{\Lambda}\right]$ the conditional expectation of a random variable $g$ on $\Omega$ with respect to the sub- $\sigma$-algebra $\mathbb{Q}_{\Lambda}$.

If a probability $\nu$ on a $\sigma$-algebra $\mathscr{B}$ is absolutely continuous with respect to another probability $\nu^{\prime}$ on $\mathscr{B}$, we write $\nu \ll \nu^{\prime}$.

We say that $\mu$ is a local Markov field (resp. Markov field) if $\left(X_{t}\right)$ is a local Markov process (resp. Markov process) under $\mu$.

The main result of the paper is

THEOREM 1. Let $T=\mathbf{R}$ or $\mathbf{Z},(E, \mathcal{E})$ a standard Borel space. Let $\mu$ be a local Markov field on $(\Omega, Q)$ such that

(i) For every $t \in T$, there exist $t^{\prime}<t<t^{\prime \prime}$ in $T$ and $\sigma$-finite measures $\nu_{t^{\prime}}$, $\nu_{t}$, $\nu_{t^{\prime \prime}}$ on $(E, \mathcal{E})$ such that

$$
\mu_{t^{\prime}, t, t^{\prime \prime}} \ll \nu_{t^{\prime}} \otimes \nu_{t} \otimes \nu_{t^{\prime \prime}}
$$

(ii) $\mathbb{Q}_{\infty}$ (resp. $\left.\mathbb{Q}_{-\infty}\right)$ is trivial for $\mu$.

Then $\mu$ is a Markov process.

We give the proof for the case $T=\mathbf{R}$ (the case $T=\mathbf{Z}$ is similar) under the hypothesis $\mathbb{Q}_{\infty}$ trivial (for the case $\mathcal{Q}_{-\infty}$ trivial, we use the additional remark that the Markov property is symmetric with respect to the direction of time).

Proof. Let $t \in T, t^{\prime}<t<t^{\prime \prime}, \nu_{t}, \nu_{t^{\prime}}, \nu_{t^{\prime \prime}}$ as in (i), let $k$ be a positive integer greater than $t^{\prime \prime}, S=\left\{t^{\prime}, t, t^{\prime \prime}, k, k+1, k+2, \ldots\right\}$

(a) On $(E, \mathcal{E})^{S}=(E, \mathcal{E})^{t} \times(E, \mathcal{E})^{S \backslash t}$ we have $\mu_{S} \ll \nu_{t} \otimes \mu_{S \backslash t}$ :

Let $(E, \mathcal{E})^{S}=(E, \mathcal{E})^{\{t\}} \times(E, \mathcal{E})^{\left\{t^{\prime}, t^{\prime \prime}\right\}} \times(E, \mathcal{E})^{\{k, k+1, \ldots\}}$,

$$
(E, \mathcal{E})^{S}=(X, \mathscr{X}) \times(Y, \mathscr{Y}) \times(Z, \mathscr{Z}) .
$$

Consider the measure $\mu_{\left\{t^{\prime}, t, t^{\prime \prime}\right\}}$ on $X \times Y=E^{\{t\}} \times E^{\left\{t^{\prime}, t^{\prime \prime}\right\}}$ and let $\mu^{\left(x^{\prime}, x^{\prime \prime}\right)}(F)$, for $\left(x_{t^{\prime}}, x_{t^{\prime \prime}}\right) \in Y, F \in \mathscr{X}$ be the transition probability on $Y \times \mathcal{X}$ such that

$$
\mu_{\left\{t^{\prime}, t, t^{\prime \prime}\right\}}=\int_{Y} \mu^{\left(x_{t^{\prime}}, x_{t^{\prime \prime}}\right)} d \mu_{\left\{t^{\prime}, t^{\prime \prime}\right\}}\left(x_{t^{\prime}}, x_{t^{\prime \prime}}\right)
$$

(cf. [3, Proposition V.4.4, p. 183]).

And similarly on $X \times(Y \times Z)$ let $\mu^{\left(x_{t}^{\prime}, x^{\prime \prime}, x_{k}, x_{k+1}, \cdots\right)}(F)$, for $\left(x_{t^{\prime}}, x_{t^{\prime \prime}}, x_{k}\right.$, $\left.x_{k+1}, \ldots\right) \in Y \times Z, F \in \mathcal{X}$ be the transition probability on $(Y \times Z) \times \mathscr{X}$ such that

$$
\mu_{S}=\int_{Y \times Z} \mu^{\left(x_{t}, x_{t}^{\prime \prime}, x_{k}, x_{k+1}, \ldots\right)} d \mu_{S \backslash t}\left(x_{t^{\prime}}, x_{t^{\prime \prime}}, x_{k}, \ldots\right) .
$$

By local Markov property (0), we have

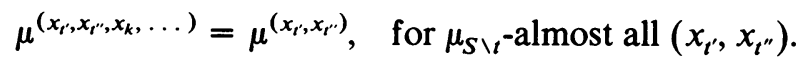

But by (i), we have $\mu_{t^{\prime}, t, t^{\prime \prime}} \ll \nu_{t^{\prime}} \otimes \nu_{t} \otimes \nu_{t^{\prime \prime}}$ and $\mu_{t^{\prime}, t^{\prime \prime}} \ll \nu_{t^{\prime}} \otimes \nu_{t^{\prime \prime}}$, so relation (2) implies

$$
\mu^{\left(x_{t^{\prime}}, x_{t^{\prime \prime}}\right)} \ll \nu_{t}, \text { for } \mu_{t^{\prime}, t^{\prime \prime}} \text {-almost all }\left(x_{t^{\prime}}, x_{t^{\prime \prime}}\right) .
$$

Comparing (4) and (5), we deduce that

$$
\mu^{\left(x_{t}, x_{t^{\prime}}, x_{k}, \ldots\right)} \ll \nu_{t}, \text { for } \mu_{S \backslash t^{-}} \text {-almost all }\left(x_{t^{\prime}}, x_{t^{\prime \prime}}, x_{k}, \ldots\right) .
$$


Combining this relation and (3), we obtain

$$
\mu_{S} \ll \nu_{t} \otimes \mu_{S \backslash t}
$$

(b) We have $\mathbb{Q}_{t}=\lim _{n \rightarrow \infty} \mathbb{Q}_{t} \vee\left(\bigvee_{m \geqslant n} \mathbb{Q}_{m}\right)$ : Let $(X, \mathcal{X})=(E, \mathcal{E})^{\{t\}},(W$, $\mathscr{W})=(E, \mathcal{E})^{S \backslash\{t\}}$, then $(E, \mathcal{E})^{S}=(X, \mathscr{X}) \times(W, \mathscr{W})$.

Let $\mathscr{G}_{n}=\bigvee_{m \geqslant n} \mathbb{Q}_{m}$, for $n \geqslant k$, we shall show that $\lim _{n \rightarrow \infty} \mathbb{Q}_{t} \vee \mathfrak{B}_{n}=\mathbb{Q}_{t}$, $\mu_{S}$-a.e. But we remark that $-\mathbb{Q}_{t}=\mathscr{X},-\mathscr{B}_{n} \subset \mathcal{W}, \forall n \geqslant k$ and $\lim _{n \rightarrow \infty} \mathscr{B}_{n}$ $=\{\varnothing, \Omega\}, \mu_{S \backslash t}$-a.e. $-\mathcal{X}$ and $\mathscr{W}$ are independent with respect to $\nu_{t} \otimes \mu_{S \backslash t}$. Therefore

$$
\lim _{n} \mathbb{Q}_{t} \vee \mathscr{B}_{n}=\mathbb{Q}_{t}, \quad \nu_{t} \otimes \mu_{S \backslash t} \text {-a.e. }
$$

and also $\mu_{s}$-a.e. since $\mu_{S} \ll \nu_{t} \otimes \mu_{S \backslash t}$.

(c) Now let $f \geqslant 0, \mathbb{Q}_{[\alpha, \beta]}$-measurable with $t \leqslant \alpha \leqslant \beta$, then (1) is satisfied:

Let $n>\beta$ with $n$ positive integer, by local Markov property (0), we have

$$
\begin{aligned}
E_{\mu}\left[f \mid \mathscr{Q}_{1-\infty, t] \cup[n,+\infty}\right] & =E_{\mu}\left[f \mid \mathbb{Q}_{\{t, n\}}\right] \\
& =E_{\mu}\left[f \mid \mathbb{Q}_{t} \vee \mathbb{Q}_{\{n, n+1, \ldots\}}\right]
\end{aligned}
$$

So by (b) and martingale theorem, we have

$$
\lim _{n \rightarrow \infty} E_{\mu}\left[f \mid \mathbb{Q}_{[-\infty, t[\cup[n,+\infty l}\right] \rightarrow E_{\mu}\left[f \mid \mathbb{Q}_{t}\right]
$$

Taking the conditional expectation with respect to $Q_{1-\infty, t]}$ of the both sides, we obtain

$$
E_{\mu}\left[f \mid \mathscr{Q}_{-\infty, t}\right]=E\left[f \mid \mathscr{Q}_{t}\right], \quad \mu \text {-a.e. }
$$

(d) From (c) we deduce (1) since the $\mathbb{Q}_{[\alpha, \beta]}, t \leqslant \alpha \leqslant \beta$, generate $\mathbb{Q}_{\{t, \infty[}$.

REMARK. It is well known that the conditions $\mathscr{G}_{n} \searrow\{\varnothing, \Omega\}$ do not imply $Q_{t} \vee \mathscr{B}_{n} \searrow Q_{t}$ without extra hypotheses.

\section{Application to statistical mechanics and one dimensional field theory.}

Local Markov SPECifications. Let $T=\mathbf{R}$ or $\mathbf{Z},(E, \mathcal{E})$ be a standard Borel space, $(\Omega, \mathcal{Q})=(E, \mathcal{E})^{T}$.

We call local Markov specifications (see [2]) the given of a family $\pi=$ $\left(\pi_{[a, b]}\right)_{a<b}$ of transition probabilities $\pi_{[a, b]}(\omega, A)(\omega \in \Omega, A \in \mathbb{Q})$ such that

(i) For every $t \in T$ there exist $a<t<b$, a $\sigma$-finite measure $\nu_{t}$ on $(E, \mathcal{E})$ such that the restriction of $\pi_{[a, b]}(\omega, \cdot)$ to $\mathbb{Q}_{t}$ is absolutely continuous with respect to $\nu_{t}, \forall \omega \in \Omega$.

(ii) $\pi_{[a, b]}(\cdot, A)$ is $\mathbb{Q}_{-\infty, a] \cup[b,+\infty[}$-measurable, $\forall A \in \mathbb{Q}$.

$\pi_{[a, b]}(\cdot, A)$ is $\mathbb{Q}_{a, b}$-measurable, for $A \in \mathbb{Q}_{[a, b]}$.

$\pi_{[a, b]}(\cdot, A)=1_{A}(\cdot)$ for $A \in \mathbb{Q}_{1-\infty, a] \cup[b,+\infty[}$.

(iii) If $[a, b] \subset[c, d]$, we have $\pi_{[c, d]} \pi_{[a, b]}=\pi_{[c, d]}$ where

$$
\pi_{[c, d]} \pi_{[a, b]}(\omega, A)=\int_{\Omega} \pi_{[c, d]}\left(\omega, d \omega^{\prime}\right) \pi_{[a, b]}\left(\omega^{\prime}, A\right), \quad \forall A \in \mathbb{Q}, \forall \omega \in \Omega .
$$

We call local Markov field (or Gibbs state) specified by $\pi$ every probability $\mu$ on $(\Omega, Q)$ satisfying

$$
E_{\mu}\left[A \mid \mathbb{Q}_{-\infty, a] \cup[b,+\infty l}\right](\omega)=\pi_{[a, b]}(\omega, A), \quad \mu \text {-a.e., } \forall A \in \mathbb{Q}_{[a, b]}
$$


The set $\mathcal{G}(\pi)$ of all local Markov fields specified by $\pi$ is a convex set which is possibly empty.

A local Markov field $\mu \in \mathcal{G}(\pi)$ is extremal iff

$$
\bigcap_{n} \bigvee_{|t|>n} Q_{t}=\{\varnothing, \Omega\}, \quad \mu \text {-a.e. (cf. [2]). }
$$

Theorem 1 implies

ThEOREM 2. Let $\left(\pi_{[a, b]}\right)_{a<b}$ be local Markov specifications. Every extremal point of $\mathcal{G}(\pi)$ has the Markov property.

Remarks. (1) When $T=\mathrm{Z}, E$ countable, this result implies Theorem 6 of F. Spitzer (cf. [5]).

(2) This result also implies Theorem 3.9 of G. Royer and M. Yor (cf. [4]).

(3) It should be interesting to know whether similar results hold when $T=\mathbf{Z}^{d}$ or $\mathbf{R}^{d}$ with $d \geqslant 2$.

(4) The characterization of Gibbs states of $\mathcal{G}(\pi)$ possessing the Markov property (other than extremal Gibbs states) is given in [1].

\section{BIBLIOGRAPHY}

1. N. Dang-Ngoc and M. Yor, Champs Markoviens et mesures de Gibbs sur $\mathbf{R}$ (to appear).

2. H. Föllmer, Phase transition and Martin boundary, Séminaire Probabilités Strasbourg IX, Lecture Notes in Math., Springer-Verlag, Berlin and New York, 1976.

3. J. Neveu, Base mathématique du calcul des probabilités, Masson et Cie., Paris, 1970.

4. G. Royer and M. Yor, Représentation intégrale de certaines mesures quasi-invariantes sur C(R); mesures extrémales et propriété de Markov, Ann. Inst. Fourier 26 (1976), 7-24.

5. F. Spitzer, Phase transition in one-dimensional nearest neighbor systems, J. Functional Analysis 20 (1975), 240-254.

Département de Mathématiques, Université de l'Etat à Mons, 7000-Mons, Belgique Département de Mathématiques, Université de Paris, 75230 Paris, Cedex 05, France 